S. Kobayashi

Nagoya Math. J.

Vol. 101 (1986), 37-54

\title{
HOMOGENEOUS VECTOR BUNDLES AND STABILITY
}

\author{
SHOSHICHI KOBAYASHI*)
}

\section{§1. Introduction}

In $[5,6,7]$ I introduced the concept of Einstein-Hermitian vector bundle. Let $E$ be a holomorphic vector bundle of rank $r$ over a complex manifold $M$. An Hermitian structure $h$ in $E$ can be expressed, in terms of a local holomorphic frame field $s_{1}, \cdots, s_{r}$ of $E$, by a positive-definite Hermitian matrix function $\left(h_{i j}\right)$ defined by

$$
h_{i j}=h\left(s_{i}, s_{j}\right) .
$$

Then the Hermitian connection form and its curvature form are given by

$$
\begin{aligned}
\omega_{j}^{i} & =\sum h^{i \bar{k}} d^{\prime} h_{j \bar{k}}, \\
\Omega_{j}^{i} & =d^{\prime \prime} \omega_{j}^{i} .
\end{aligned}
$$

In terms of a local coordinate system $z^{1}, \cdots, z^{n}$ of $M$, we can write

$$
\Omega_{j}^{i}=\sum R_{j \alpha \bar{\beta}}^{i} d z^{\alpha} \wedge d \bar{z}^{\beta} .
$$

Given an Hermitian metric

$$
g=2 \sum g_{\alpha \bar{\beta}} d z^{\alpha} d \bar{z}^{\beta}
$$

on $M$, we define the $g$-trace $K$ of the curvature of $(E, h)$ by setting

$$
K_{j}^{i}=\sum g^{\alpha \bar{B}} R_{j \alpha \bar{\beta}}^{i} .
$$

Then $K$ is a field of endomorphisms of $E$ with components $K_{j}^{i}$. We say that $(E, h, M, g)$ is an Einstein-Hermitian vector bundle if

$$
K=\varphi I_{E}, \quad \text { i.e., } \quad K_{j}^{i}=\varphi \delta_{j}^{i},
$$

where $\varphi$ is a (real) function on $M$ and $I_{E}$ is the identity endomorphism of $E$.

Received December 26, 1983.

*) Partially supported by NSF Grant MCS-8200235. This work was done while the author was a guest at Ecole Polytechnique and at the University of Bonn (SFB) in the spring of 1983. 
In $[6,7]$ I obtained the following differential geometric criterion for stability, (see Lübke [8] for a simpler proof).

(1.1) Theorem. Let $M$ be a compact complex manifold with an ample line bundle $H$ and $g$ a Kähler metric on $M$ whose Kähler form represents the Chern class of $H$. Let $E$ be a holomorphic vector bundle over $M$ and $h$ an Hermitian structure in $E$. If $(E, h, M, g)$ is an Einstein-Hermitian vector bundle, then

(a) it is $H$-semistable in the sense of Mumford-Takemoto;

(b) it is a direct sum of $\mathrm{H}$-stable Einstein-Hermitian vector bundles $\left(E_{1}, h_{1}, M, g\right), \cdots,\left(E_{q}, h_{q}, M, g\right)$ with irreducible holonomy group;

(c) $\mu\left(E_{1}\right)=\cdots=\mu\left(E_{q}\right)=\mu(E)$, where $\mu(E)$ denotes the degree-rank ratio of $E$ defined by $\mu(E)=c_{1}(E) c_{1}(H)^{n-1} / \operatorname{rank}(E), n=\operatorname{dim} M$.

If $E$ is a homogeneous vector bundle over a homogeneous algebraic manifold $M=G / G_{0}$ of a compact Lie group $G$ and if the isotropy subgroup $G_{0}$ is irreducible on the fibre $E_{o}$ of $E$ at the origin $o \in M$, then $E$ with any $G$-invariant Hermitian structure $h$ is an Einstein-Hermitian vector bundle. From (a) of $(1,1)$ it follows that $E$ is $H$-semistable for any ample line bundle $H$. In order to see whether $E$ is indeed $H$-stable or not, we study the holonomy group of $E$ in Section 2 . In Section 3 we give a differential geometric proof to the theorem of Ramanan [11] and Umemura [13] that every irreducible homogeneous vector bundle over a Kähler $C$ space $M$ (of H. C. Wang) is $H$-stable for any ample line bundle $H$. In Section 5, as an application we show that the null correlation bundles over $P_{2 n+1}$ are Einstein-Hermitian vector bundles with irreducible holonomy group (and hence, they are $H$-stable-a well known fact). Our approach to null correlation bundles is through complex contact structures (see §4). In Section 6 we construct example of stable Einstein-Hermitian bundle using complex contact structures.

\section{§ 2. Holonomy and automorphisms of Hermitian vector bundles}

Let $(E, h)$ be an Hermitian vector bundle over a complex manifold $M$. If $c=c(t), 0 \leqq t \leqq 1$, is a piecewise smooth curve in $M$, the parallel transport $\tau_{c}$ along $c$ gives an isometry between the fibres $E_{c(0)}$ and $E_{c(1)}$. Fixing a point $o$ of $M$ and considering all closed curves $c$ from $o$ to $o$, we obtain a group $\Psi$ of automorphisms of the fibre $E_{o}$ given by parallel transports $\tau_{c}$. This group $\Psi$ is called the holonomy group of $(E, h)$. We decompose 
the fibre $E_{o}$ into an orthogonal direct sum of $\Psi$-invariant subspaces:

$$
E_{o}=E_{o}^{0}+E_{o}^{1}+\cdots+E_{o}^{k},
$$

where $\Psi$ fixes $E_{o}^{0}$ elementwise (i.e., acts trivially on $E_{o}^{0}$ ) and acts irreducibly on $E_{o}^{1}, \cdots, E_{o}^{k}$. By transporting $E_{o}$ parallelly, we extend the decomposition (2.1) to a global decomposition of $E$. Thus,

$$
E=E^{0}+E^{1}+\cdots+E^{k} .
$$

This decomposition is not only orthogonal but also holomorphic since the Hermitian connection $D$ (as covariant differentiation) is of the form $D=$ $D^{\prime}+d^{\prime \prime}$. (In fact, if $s=s_{0}+s_{1}+\cdots+s_{k}$ is a local holomorphic section of $E$ and $s_{0}, s_{1}, \cdots, s_{k}$ are local $C^{\infty}$ sections of $E^{0}, E^{1}, \cdots, E^{k}$, respectively, then from $D^{\prime \prime} s=d^{\prime \prime} s=0$ we obtain $d^{\prime \prime} s_{i}=D^{\prime \prime} s_{i}=0$ for all $i$, showing that $s_{0}, s_{1}, \cdots, s_{k}$ are holomorphic sections of $E$. This means that $E^{0}, E^{1}$, $\cdots, E^{k}$ are holomorphic subbundles of $E$ ).

Let $G$ be a group of automorphisms of the Hermitian vector bundle $(E, h)$. Each element $f$ of $G$ induces a holomorphic transformation $\bar{f}$ of $M$. Since $f$ preserves the connection of $E$, for each curve $c=c(t), 0 \leq t \leq 1$, of $M$ we have

$$
\tau_{\bar{f} \circ c} \circ f=f \circ \tau_{c},
$$

where both sides are considered as transformations $E_{c(0)} \rightarrow E_{c(1)}$. If $c$ is a closed curve starting from $o$ and if $\bar{f}(o)=o$, then both sides are automorphisms of the fibre $E_{o}$.

Let $G_{0}$ be the isotropy subgroup of $G$ at $o$, i.e., $G_{0}=\{f \in G ; f(o)=o\}$. From (2.3) we obtain

$$
f \circ \tau \circ f^{-1} \in \Psi \quad \text { for } f \in G_{o} \text { and } \tau \in \Psi,
$$

i.e., $G_{o}$ normalizes the holonomy group $\Psi$.

Following the decomposition (2.1) of the fibre $E_{o}$, we can express each element $\tau$ of the holonomy group $\Psi$ by a matrix of the form

$$
A(\tau)=\left(\begin{array}{cccc}
A_{0}(\tau) & 0 & \cdots & 0 \\
0 & A_{1}(\tau) & \cdots & 0 \\
\cdot & \cdot & \cdots & 0 \\
0 & 0 & \cdots & A_{k}(\tau)
\end{array}\right) .
$$

We consider an element $X$ of the Lie algebra of $G_{0}$, i.e., $X=(d f(s) / d s)_{s=0}$, 
where $f(s)$ is a 1-parameter subgroup of $G_{0}$. Corresponding to the decomposition (2.1) of $E_{o}, X$ can be written in the following block form:

$$
X=\left(\begin{array}{cccc}
X_{00} & X_{01} & \cdots & X_{0 k} \\
X_{10} & X_{11} & \cdots & X_{1 k} \\
\cdot & \cdot & \cdots & \cdot \\
X_{k 0} & X_{k 1} & \cdots & X_{k k}
\end{array}\right)
$$

Since $f(s) \circ \tau \circ f(s)^{-1} \in \Psi$ by (2.4), the corresponding matrix has zeros off the diagonal blocks. Differentiating $f(s) \circ \tau \circ f(s)^{-1}$ at $s=0$, we obtain $f^{\prime}(0) \circ \tau-\tau \circ f^{\prime}(0)$. Hence, the corresponding matrix

$$
X \cdot A(\tau)-A(\tau) \cdot X
$$

must have zeros off the diagonal blocks. Thus,

$$
X_{i j} \cdot A_{j}(\tau)=A_{i}(\tau) \cdot X_{i j} \quad \text { for } \quad i \neq j .
$$

Since the holonomy group $\Psi$ acts trivially on $E_{o}^{0}$ and irreducibly on $E_{o}^{1}, \cdots, E_{o}^{k}$, it follows that

$$
A_{0}(\tau)=I \quad \text { for } \quad \tau \in \Psi
$$

and that the representations

(2.10) $A_{i}: \tau \longmapsto A_{i}(\tau), i=1, \cdots, k$, are irreducible.

By Schur's lemma, we have

(2.11) $X_{i j}=0$ unless the representations $A_{i}$ and $A_{j}$ are equivalent. We note that we have always $X_{0 j}=0$ and $X_{j 0}=0$ for $j=1, \cdots, k$.

If $A_{i}$ and $A_{j}$ are equivalent, by changing a basis in $E_{o}$ we may assume that $A_{i}=A_{j}$. By parallel transport of such bases in $E_{o}^{i}$ and $E_{o}^{j}$ we obtain an isomorphism between $E^{i}$ and $E^{j}$. We have established the following

(2.12) Theorem. Let $(E, h)$ be an Hermitian vector bundle over a complex manifold $M$. Let $o \in M$ and $G_{0}$ a connected Lie group of automorphisms of $(E, h)$ leaving the fibre $E_{o}$ invariant. If $G_{o}$ acts irreducibly on $E_{o}$, then

$$
(E, h)=\left(E^{\prime}, h^{\prime}\right)+\cdots+\left(E^{\prime}, h^{\prime}\right), \quad(q \text { copies, say }),
$$

where $\left(E^{\prime}, h^{\prime}\right)$ is an Hermitian vector bundle with irreducible holonomy group. 
The decomposition $E=E^{\prime}+\cdots+E^{\prime}$ in (2.12) may be written as

$$
E=E^{\prime} \otimes C^{q},
$$

where $C^{q}$ denotes the product bundle of rank $q$.

Assuming that $(E, h)$ is a direct sum of $q$ copies of $\left(E^{\prime}, h^{\prime}\right)$ with irreducible holonomy but without assuming that $G_{0}$ acts irreducibly on $E_{o}$, we shall study automorphisms $f$ of $(E, h)$. Let $f$ be an automorphism of $(E, h)$ with the induced transformation $\bar{f}$ of $M$. We set

$$
o^{\prime}=\bar{f}(o) .
$$

We denote the holonomy group of $(E, h)$ with reference point $o^{\prime}$ by $\Psi^{\prime}$. We fix a curve $a$ from $o$ to $o^{\prime}$ and assign to each loop $c$ at $o$ the loop $a \circ c \circ a^{-1}$ at $o^{\prime}$. This gives an isomorphism $\Psi \rightarrow \Psi^{\prime}$. To a basis in $E_{o}^{\prime}$ we associate the basis in $E_{o^{\prime}}^{\prime}$ obtained by parallel transport along the curve $a$. Then the corresponding elements under the identification $\Psi=\Psi^{\prime}$ have the same matrix representation.

The matrix $A(\tau)$ representing an element $\tau \in \Psi$ is of the form

$$
A(\tau)=\left(\begin{array}{cccc}
B(\tau) & 0 & \cdots & 0 \\
0 & B(\tau) & \cdots & 0 \\
\cdots & \ldots & \cdots & \cdots \\
0 & 0 & \cdots & B(\tau)
\end{array}\right)
$$

where the representation $B$ is irreducible since $\left(E^{\prime}, h^{\prime}\right)$ is assumed to have an irreducible holonomy.

An automorphism $f$ sends the fibre $E_{o}$ to the fibre $E_{o^{\prime}}$. With respect to the bases for $E_{o}$ and $E_{o}$, chosen as above, we represent $f$ by a matrix $F$ :

$$
F=\left(\begin{array}{ccc}
F_{11} & \cdots & F_{1 q} \\
\cdots & \cdots & \cdots \\
F_{q 1} & \cdots & F_{q q}
\end{array}\right),
$$

where each $F_{i j}$ is a $(p \times p)$ matrix, $p=\operatorname{rank} E^{\prime}$.

Let $\tau=\tau_{c} \in \Psi$ and $\tau^{\prime}=\tau_{\jmath_{o c}} \in \Psi^{\prime}$. From (2.3) we obtain

$$
F A(\tau)=A\left(\tau^{\prime}\right) F
$$

Hence,

$$
F_{i j} B(\tau)=B\left(\tau^{\prime}\right) F_{i j} \quad \text { for all } i, j
$$


Each $F_{i j}$ is non-singular unless $F_{i j}=0$. (For if $v$ is a vector such that $F_{i j} v=0$, then $F_{i j} B(\tau) v=0$ by (2.17), which implies that the kernel of the linear transformation $F_{i j}$ is invariant by $B(\tau), \tau \in \Psi$. Since $B$ is an irreducible representation, the kernel of $F_{i j}$ is either 0 or the whole space).

We claim next that

$$
F_{i j}=u_{i j} V \quad \text { for all } i, j,
$$

where $V$ is a $(p \times p)$ unitary matrix and $U=\left(u_{i j}\right)$ is a $(q \times q)$ unitary matrix. (It suffices to show that $F_{i j}$ is a scalar multiple of $F_{m n}$ when both $F_{i j}$ and $F_{m n}$ are non-singular. Eliminating $B\left(\tau^{\prime}\right)$ from

$$
F_{i j} B(\tau)=B\left(\tau^{\prime}\right) F_{i j} \quad \text { and } \quad F_{m n} B(\tau)=B\left(\tau^{\prime}\right) F_{m n},
$$

we obtain

$$
F_{m n}^{-1} F_{i j} B_{i j}(\tau)=B(\tau) F_{m n}^{-1} F_{i j} \quad \text { for all } \quad \tau \in \Psi
$$

Since $B$ is irreducible, $\left.F_{m n}^{-1} F_{i j}=c I\right)$.

Hence, $F$ can be written as the Kronecker product:

$$
F=V \otimes U .
$$

This corresponds to the tensor product $E=E^{\prime} \otimes C^{q}$, i.e.,

$$
f(\eta \otimes \xi)=V \eta \otimes U \xi \quad \text { for } \eta \otimes \xi \in\left(E^{\prime} \times C^{q}\right)_{o}=E_{o} .
$$

Any $(q \times q)$ unitary matrix $U$ defines an automorphism $f_{U}$ of $(E, h)$ by

$$
f_{U}(\eta \otimes \xi)=\eta \otimes U \xi \quad \text { for } \quad \eta \otimes \xi \in E^{\prime} \otimes C^{q}=E .
$$

Such an automorphism induces the identity transformation on the base manifold $M$, i.e.,

$$
\bar{f}_{U}=\mathrm{id}_{M} .
$$

Conversely, assume that $f$ is an automorphism of $(E, h)$ such that $\bar{f}=$ $\mathrm{id}_{\mu}$. By (2.3), $f$ commutes with every element of the holonomy group $\Psi$. With the notation of (2.14) and (2.15), we have

$$
F A(\tau)=A(\tau) F \quad \text { on } \quad E_{o} \text { for all } \tau \in \Psi .
$$

Hence,

$$
F_{i j} B(\tau)=B(\tau) F_{i j} \quad \text { for all } \tau \in \Psi .
$$

Since $B$ is an irreducible representation, it follows that 


$$
F_{\imath j}=u_{i j} I \quad \text { for all } i, j \text {. }
$$

This means

$$
F=I \otimes U \quad \text { on } \quad E_{o},
$$

where $I$ denotes the identity transformation of $E_{o}^{\prime}$. Now, varying the point $o$ in $M$, we obtain (2.22) on $E_{x}$ for all $x$ in $M$. Thus,

$$
F_{x}=I \otimes U_{x} \quad \text { on } \quad E_{x} .
$$

We claim that $U_{x}$ does not depend on $x$. To see this, let $c$ be a curve from $o$ to $x$. By (2.3), since $\bar{f}=\mathrm{id}_{M}$, we have

$$
f \circ \tau_{c}=\tau_{c} \circ f .
$$

Since $C^{q}$ is the product bundle with the natural flat Hermitian structure, we have

$$
\tau_{c}=\tau_{c}^{\prime} \otimes I \quad \text { on } \quad E_{o}=\left(E^{\prime} \otimes C^{q}\right)_{o},
$$

where $\tau_{c}^{\prime}$ is the parallel transport in $E^{\prime}$ along $c$ and $I$ is the obvious parallel transport in the product bundle $C^{q}$. Then

$$
\begin{aligned}
& \left(f \circ \tau_{c}\right)(\eta \otimes \xi)=f\left(\tau_{c}^{\prime} \eta \otimes \xi\right)=\tau_{c}^{\prime} \eta \otimes U_{x} \xi, \\
& \left(\tau_{c} \circ f\right)(\eta \otimes \xi)=\tau_{c}(\eta \otimes U \xi)=\tau_{c}^{\prime} \eta \otimes U \xi .
\end{aligned}
$$

By (2.23), we can conclude $U_{x}=U$.

We have thus established that for an automorphism $f$ of $(E, h)$ (2.25) $\bar{f}=\mathrm{id}_{M} \quad$ if and only if $f(\eta \otimes \xi)=\eta \otimes U \xi$ for $\eta \otimes \xi \in E^{\prime} \times C^{q}$, where $U$ is a $(q \times q)$ unitary matrix.

We shall now study automorphisms of $(E, h)$ preserving the decomposition $E=E^{\prime}+\cdots+E^{\prime}$. It follows from (2.3) that, in general, an automorphism $f$ of $(E, h)$ preserving the decomposition (2.1) at one point $o$ preserves the decomposition (2.2) globally, that is,

$$
f\left(E^{i}\right)=E^{i} \quad \text { if } \quad f\left(E_{o}^{i}\right)=E_{o^{\prime}}^{i} \quad \text { for } \quad i=0,1, \cdots, k,
$$

where $o^{\prime}=\bar{f}(o)$. Going back to the present situation where $E=E^{\prime}+\cdots$ $+E^{\prime}$, we see that if an automorphism $f$ of $(E, h)$ preserves the decomposition $E=E^{\prime}+\cdots+E^{\prime}$ by sending each factor into itself, then the unitary matrix $U$ of (2.18) must be diagonal, i.e.,

$$
u_{i j}=0 \quad \text { for } i \neq j \text {. }
$$

Denoting the restriction of $f$ to the $i$-th factor of the decomposition 
$E=E^{\prime}+\cdots+E^{\prime}$ by $f_{i}$, we write

$$
f=\left(f_{1}, \cdots, f_{q}\right), \quad f_{i} \in \operatorname{Aut}\left(E^{\prime}, h^{\prime}\right) .
$$

Writing

$$
c_{i}=u_{i i} \quad i=1, \cdots, q,
$$

set

$$
f_{i}^{\prime}=\frac{1}{c_{i}} f_{i} \quad i=1, \cdots, q .
$$

We shall show that $f_{1}^{\prime}=\cdots=f_{q}^{\prime}$. Since $f$ is given by $V \otimes U$ on $E_{o}, f_{i}$ is given by $u_{i i} V$ on $E_{o}^{\prime}$. Hence, $f_{1}^{\prime}=\cdots=f_{q}^{\prime}$ on $E_{o}^{\prime}$. Consider, for example, $g=f_{2}^{\prime-1} \circ f_{1}^{\prime}$. Then $g$ induces the identity automorphism of the fibre $E_{o}$ and the identity transformation $\bar{g}=\mathrm{id}_{M}$. Applying (2.25) to $g$, we see that $g$ is the identity automorphism of $\left(E^{\prime}, h^{\prime}\right)$. Hence, $f_{1}^{\prime}=f_{2}^{\prime}$, proving our assertion. Set $f^{\prime}=f_{1}^{\prime}=\cdots=f_{q}^{\prime}$. Thus we have established that if $f$ is an automorphism of $(E, h)$ preserving the decomposition $E=E^{\prime}+\cdots$ $+E^{\prime}$ factorwise, then

$$
f=\left(c_{1} f^{\prime}, \cdots, c_{q} f^{\prime}\right),
$$

where $f^{\prime} \in \operatorname{Aut}\left(E^{\prime}, h^{\prime}\right)$ and $\left|c_{1}\right|=\cdots=\left|c_{q}\right|=1$.

Now, we shall study the case where $f$ is an arbitrary automorphism of $(E, h)$. Let $o^{\prime}=\bar{f}(o)$. Let $U$ be the $(q \times q)$ unitary matrix given by (2.18) and $f_{U}$ the automorphism given by (2.21). From (2.20) we obtain

$$
\left(f_{U}^{-1} \circ f\right)(\eta \otimes \xi)=V_{\eta} \otimes \xi,
$$

which shows that the automorphism $f_{U}^{-1}$ of preserves the decomposition $E=E^{\prime}+\cdots+E^{\prime}$ at $o$ and hence globally. Then $f_{U}^{-1} \circ f$ must be of the form (2.29):

$$
f_{U}^{-1} \circ f=\left(c_{1} f^{\prime}, \cdots, c_{q} f^{\prime}\right) .
$$

Let $C$ be the diagonal unitary matrix with diagonal entries $c_{1}, \cdots, c_{q}$. Then $(2.30)$ can be rewritten as

$$
\left(f_{U}^{-1} \circ f\right)(\eta \otimes \xi)=\left(f^{\prime} \eta \otimes C \xi\right) .
$$

Hence,

$$
f(\eta \otimes \xi)=\left(f^{\prime} \eta \otimes U C \xi\right),
$$


or

$$
f=f^{\prime} \otimes f_{U C} .
$$

Absorbing $C$ into $U$, we write $U$ for $U C$. Thus, every automorphism $f$ of $(E, h)$ is of the form

$$
f=f^{\prime} \otimes f_{U}
$$

where $f^{\prime}$ is an automorphism of $\left(E^{\prime}, h^{\prime}\right)$ and $f_{U}$ is the multiplication by a $(q \times q)$ unitary matrix $U$ as in (2.21). In other words,

$$
f(\eta \otimes \xi)=f^{\prime} \eta \otimes U \xi \quad \eta \otimes \xi \in E^{\prime} \otimes C^{q} .
$$

This means that the group homomorphism

$$
\operatorname{Aut}\left(E^{\prime}, h^{\prime}\right) \times U(q) \longrightarrow \operatorname{Aut}(E, h)
$$

sending $\left(f^{\prime}, U\right)$ to $f^{\prime} \otimes f_{U}$ is surjective. Its kernel consists of $\left(\mu_{u},(1 / u) I\right)$, where $u$ is a complex number with $|u|=1$ and $\mu_{u}: E^{\prime} \rightarrow E$ denotes the multiplication by $u$.

Summarizing what we have proved, we state

(2.33) Theorem. Let $\left(E^{\prime}, h^{\prime}\right)$ be an Hermitian vector bundle over $M$ with irreducible holonomy group and $\boldsymbol{C}^{q}$ be the product bundle of rank $q$ over $M$ with the natural flat Hermitian structure. Let $E=E^{\prime} \otimes C^{q}$ and let $h$ be the naturally induced Hermitian structure in $E$. Then the automorphism groups Aut $(E, h)$ and Aut $\left(E^{\prime}, h^{\prime}\right)$ are related by the following exact sequence:

$$
1 \longrightarrow U(1) \stackrel{i}{\longrightarrow} \operatorname{Aut}\left(E^{\prime}, h^{\prime}\right) \otimes U(q) \stackrel{j}{\longrightarrow} \operatorname{Aut}(E, h) \longrightarrow 1,
$$

where

$$
j\left(f^{\prime}, U\right)=f^{\prime} \otimes f_{L^{\prime}}, \quad i(u)=\left(\mu_{u}, \frac{1}{u} I\right) .
$$

(In the definitions of $j$ and $i$ above, $f_{U}$ is the multiplication by $U \in$ $U(q)$ as defined in (2.21), and $\mu_{u}$ is also the multiplication by a scalar $u$ ).

The natural projections from Aut $\left(E^{\prime}, h^{\prime}\right) \times U(q)$ to Aut $\left(E^{\prime}, h^{\prime}\right)$ and $U(q)$ induce homomorphisms

$$
\begin{aligned}
& \alpha: \operatorname{Aut}(E, h) \longrightarrow P \operatorname{Aut}\left(E^{\prime}, h^{\prime}\right):=\operatorname{Aut}\left(E^{\prime}, h^{\prime}\right) /\left\{\mu_{u} ; u \in U(1)\right\}, \\
& \beta: \operatorname{Aut}(E, h) \longrightarrow P U(q):=U(q) /\{u I ; u \in U(1)\},
\end{aligned}
$$


(where $P$ stands for "projective").

Then

$$
\begin{aligned}
& \operatorname{Ker} \alpha=\left\{f_{U} ; U \in U(q)\right\} \approx U(q), \\
& \operatorname{Ker} \beta=\left\{f^{\prime} \otimes I ; f^{\prime} \in \operatorname{Aut}\left(E^{\prime}, h^{\prime}\right)\right\} \approx \operatorname{Aut}\left(E^{\prime}, h^{\prime}\right) .
\end{aligned}
$$

\section{$\S 3$. Homogeneous Hermitian vector bundles}

Let $G$ be a connected, compact semi-simple Lie group, $T$ a toral subgroup of $G$, and $C(T)$ the centralizer of $T$ in $G$. Then $G / C(T)$ is a simply connected, compact homogeneous Kähler manifold, and conversely, (Wang [14] and Borel [3]).

We need the following simple lemma.

(3.1) Lemma. Let $G$ and $C(T)$ be as above and assume that $C(T)$ contains no simple factor of $G$. Let $P U(n)=U(n) /\{u I ; u \in U(1)\}$ denote the projective unitary group. If $\rho: G \rightarrow P U(n)$ is a representation of $G$, then its restriction to $C(T)$ is always a reducible representation.

Proof. We may assume that $\rho$ is non-trivial, i.e., $\rho(G) \neq\{I\}$. Let $T^{\prime}=\rho(T)$. Then $T^{\prime}$ is a non-trivial toral subgroup of $P U(n)$. (If $T^{\prime}$ is trivial so that $T \subset \operatorname{Ker} \rho$, then $C(T)$ must contain all simple factors of $G$ which are not in $\operatorname{Ker} \rho$ ). Let $C\left(T^{\prime}\right)$ be the centralizer of $T^{\prime}$ in $P U(n)$. Since $T^{\prime}$ may be considered as a subgroup of the diagonal subgroup of $P U(n)$, its centralizer $C\left(T^{\prime}\right)$ is of the form $P\left(U\left(n_{1}\right) \times \cdots \times U\left(n_{k}\right)\right)=\left(U\left(n_{1}\right)\right.$ $\left.\times \cdots \times U\left(n_{k}\right)\right) /\{u I ; \quad u \in U(1)\}$, where $n=n_{1}+\cdots+n_{k}$. By $U\left(n_{1}\right) \times \cdots$ $\times U\left(n_{k}\right)$, we mean the subgroup of $U(n)$ of the form

$$
\left(\begin{array}{ccc}
U\left(n_{1}\right) & & \\
0 & \ddots & 0 \\
& & U\left(n_{k}\right)
\end{array}\right) .
$$

Since $\rho(C(t)) \subset C\left(T^{\prime}\right)$, Lemma follows immediately.

Q.E.D.

Let $(E, h)$ be an Hermitian vector bundle over a complex manifold $M$. Let $G$ be a group of automorphism of $(E, h)$. Let $o \in M$ and $G_{0}$ the subgroup of $G$ consisting of automorphisms leaving the fibre $E_{o}$ invariant. If $G_{0}$ acts irreducibly on $E_{o}$, then $(E, h)$ is of the form $\left(E^{\prime}, h^{\prime}\right) \otimes C^{q}$, (see (2.12)). In the preceding section, we defined a homomorphism $\beta$ : Aut $(E, h)$ $\rightarrow P U(q)$. If $G$ is connected, compact and semi-simple and if $G_{0}=C(T)$, the centralizer of a toral subgroup $T$ of $G$, then (3.1) implies that $\beta$ : $G_{0}$ $\rightarrow P U(q)$ is a reducible representation. Let $S \subset C^{q}$ be a subspace invariant 
by $\beta\left(G_{0}\right)$. Then $\left(E^{\prime} \otimes S\right)_{0}$ is a subspace of $E_{o}=\left(E^{\prime} \otimes C^{q}\right)_{0}$ invariant by $G_{0}$. Since we assumed that $G_{0}$ acts irreducibly on $E_{o}$, this contradiction means that we must have $q=1$, i.e., $E=E^{\prime}$ in (2.12). We have shown the following

(3.2) Proposition. Let $(E, h)$ be an Hermitian vector bundle over a complex manifold $M$. Let $G$ be a connected, compact, semi-simple Lie group of automorphisms of $(E, h)$. Let $o \in M$ and $G_{0}$ the subgroup of $G$ consisting of automorphisms leaving the fibre $E_{o}$ invariant. If $G_{0}$ acts irreducibly on $E_{o}$ and if $G_{0}$ is of the form $C(T)$ for some toral subgroup $T$ of $G$, then the holonomy group of $(E, h)$ is irreducible.

We prove now the following

(3.3) Theorem. Let $E$ be a holomorphic vector bundle over a compact complex manifold $M$ with an ample line bundle $H$. Let $G$ be a connected, compact Lie group of automorphisms of E acting transitively on $M$. Assume that the isotropy subgroup $G_{0}$ of $G$ at a point $o \in M$ acts irreducibly on the fibre $E_{o}$ at $o$. Then

(1) There exists a G-invariant Hermitian structure in $E$ and $a G$ invariant Kähler metric $g$ on $M$ whose Kähler form represents the Chern class $c_{1}(H)$ of $H$, and $(E, h, M, g)$ is an Einstein-Hermitian vector bundle.

(2) Moreover,

$$
(E, h)=\left(E^{\prime}, h^{\prime}\right)+\cdots+\left(E^{\prime}, h^{\prime}\right)=\left(E^{\prime}, h^{\prime}\right) \otimes C^{q},
$$

where $\left(E^{\prime}, h^{\prime}\right)$ is an Einstein-Hermitian vector bundle over $(M, g)$ with irreducible holonomy group. The vector bundle $E^{\prime}$ is H-stable in the sense of Mumford-Takemoto.

Proof. (1). Averaging an arbitrary Hermitian structure of $E$ by the action of $G$, we obtain a $G$-invariant Hermitian structure $h$ in $E$. Similarly, we start with a Kähler metric on $M$ whose Kähler form represents $c_{1}(H)$. Averaging it by the action of $G$, we obtain a $G$-invariant Kähler metric $g$ on $M$. Since $G$ is connected, the Kähler form of $g$ still represents $c_{1}(H)$.

Since the $g$-trace $K$ of the curvature $R$ is invariant by $G$ and since $G_{0}$ is irreducible on $E_{o}$, the endomorphism $K_{o}$ must be a scalar multiple of the identity transformation of $E_{o}$.

(2). The first assertion follows from (2.12). The second assertion 
follows from (1.1).

(3.4) Theorem. In (3.3), assume further that $G$ is semisimple and $G_{0}$ is the centralizer $C(T)$ of a toral subgroup $T$ of $G$. Then $(E, h)$ is an Einstein-Hermitian vector bundle over $(M, g)$ with irreducible holonomy group and $E$ is $H$-stable for any ample line bundle $H$ over $M$.

Proof. This follows from (3.2) and (3.3). Q.E.D.

We note that the second assertion in (3.4) is equivalent to the following theorem of Ramanan [11] and Umemura [13].

(3.5) Theorem. Let $L$ be a simply connected, semisimple complex Lie group and $P$ a parabolic subgroup simple factor. Let $\rho$ be a finite dimensional irreducible representation of $P$. Then the homogeneous vector bundle $E_{\rho}$ over $M=L / P$ defined by $\rho$ is $H$-stable for any ample line bundle $H$ over $M$.

We can pass from (3.4) to (3.5) by letting $L$ to be the complexification of $G$.

\section{§4. Complex contact structures}

Let $M$ be a complex manifold of dimension $2 n+1$. A complex contact structure on $M$ is given by an open cover $\left\{U_{i}\right\}$ and a system of holomorphic 1-forms $\left\{\omega_{i}\right\}$ such that

(a) Each $\omega_{i}$ is a holomorphic 1-form defined on $U_{i}$ and vanishes nowhere;

(b) The holomorphic $(2 n+1)$-form $\omega_{i} \wedge\left(d \omega_{i}\right)^{n}$ vanishes nowhere;

(c) If $U_{i} \cap U_{j}$ is non-empty, there exists a (nowhere-vanishing) holomorphic function $f_{i j}$ on $U_{i} \cap U_{j}$ such that $\omega_{i}=f_{i j} \omega_{j}$ on $U_{i} \cap U_{j}$.

Two complex contact structures $\left\{U_{i}, \omega_{i}\right\}$ and $\left\{V_{\lambda}, \theta_{\lambda}\right\}$ are considered to be equivalent if $\omega_{i}=a_{i \lambda} \theta_{\lambda}$ on $U_{i} \cap V_{\lambda}$ with a suitable holomorphic function $a_{i \lambda}$. We are, of course, interested in equivalence classes of complex contact structures.

Given a complex contact structure $\left\{U_{i}, \omega_{i}\right\}$ on $M$, we obtain a holomorphic subbundle $E$ of rank $2 n$ of the tangent bundle TM:

$$
E=\left\{X \in T M ; \omega_{i}(X)=0\right\} \text {. }
$$

Let $F$ be the line bundle defined by the transition functions $\left\{f_{i j}\right\}$ above. Then we have an exact sequence 


$$
0 \longrightarrow E \longrightarrow T M \longrightarrow F \longrightarrow 0 \text {. }
$$

Since

$$
\omega_{i} \wedge\left(d \omega_{i}\right)^{n}=\left(f_{i j}\right)^{n+1} \omega_{j} \wedge\left(d \omega_{j}\right)^{n},
$$

it follows that the determinant bundle $\operatorname{det}(T M)=\Lambda^{2 n+1} T M$ is defined by the transition functions $\left\{f_{i j}^{n+1}\right\}$. Hence,

$$
c_{1}(M)=(n+1) c_{1}(F) .
$$

From (4.2) we have

(4.5) $1+c_{1}(M)+c_{2}(M)+\cdots=\left(1+c_{1}(F)\right)\left(1+c_{1}(E)+c_{2}(E)+\cdots\right)$.

In particular,

$$
c_{1}(E)=n c_{1}(F) .
$$

From (c), we obtain

$$
d \omega_{i}=f_{i j} d \omega_{j}+d f_{i j} \wedge \omega_{j} .
$$

Since $\omega_{j}=0$ on $E$, (4.7) implies that we have a skew-symmetric blinear form $\left\{d \omega_{i}\right\}$ on $E$ with values in $F$ :

$$
\left\{d \omega_{i}\right\}: E \times E \longrightarrow F .
$$

Condition (b) implies that this bilinear form is everywhere non-degenerate on $E$. In particular, it defines an isomorphism

$$
E \approx E^{*} \times F .
$$

This imposes further conditions on Chern classes of $M$.

For complex contact structures, see Kobayashi [4], Boothby [1,2] and Wolf [15]]. The compact simply connected homogeneous complex contact manifolds were classified by Boothby. They are 2-sphere bundles over compact simply connected quaternionic symmetric spaces. This natural correspondence between the compact simply connected homogeneous complex contact manifolds and the compact connected quaternionic symmetric spaces was explained by Wolf. In today's terminology, it is nothing but the twistor construction, (see Salamon [12]).

\section{$\S 5$. Null correlation bundles}

We shall first describe a natural complex contact structure on the 
complex projective space $P_{2 n+1}$ of dimension $2 n+1$. Let $z^{0}, z^{1}, \cdots, z^{2 n+1}$ be a natural coordinate system in $C^{2 n+2}$, which will be taken as a homogeneous coordinate system for $P_{2 n+1}$. On $C^{2 n+2}-\{0\}$ (considered as a principal $C^{*}$-bundle over $P_{2 n+1}$ ) we consider the following holomorphic 1form:

$$
\omega=z^{0} d z^{1}-z^{1} d z^{0}+\cdots+z^{2 n} d z^{2 n+1}-z^{2 n+1} d z^{2 n} .
$$

Let $\left\{U_{i}\right\}$ be an open cover of $P_{2 n+1}$ with a system of local holomorphic sections $s_{i}$ of the bundle $C^{2 n+2}-\{0\}$ over $U_{i}$. Setting

$$
\omega_{i}=s_{i}^{*} \omega \text {, }
$$

we obtain a complex contact structure $\left\{U_{\imath}, \omega_{i}\right\}$ on $P_{2 n+1}$.

We identify the complex vector space $C^{2 n+2}$ with the quaternionic vector space $\boldsymbol{H}^{n+1}$ by setting

$$
q^{0}=z^{0}+z^{1} j, \cdots, q^{n}=z^{2 n}+z^{2 n+1} j .
$$

The identification $\boldsymbol{C}^{2 n+2}=\boldsymbol{H}^{n+1}$ induces a fibering

$$
P_{2 n+1} \longrightarrow P_{n} \mathrm{H}
$$

whose fibers are complex projective lines in $P_{2 n+1}$. In order to understand this fibering group-theoretically, we consider $P_{2 n+1}$ as a homogeneous space of the symplectic group $S p(n+1)$ rather than the special unitary group $S U(2 n+2)$. Thus,

$$
P_{2 n+1}=S p(n+1) / S p(n) \times T^{1} \longrightarrow P_{n} \boldsymbol{H}=S p(n+1) / S p(n) \times S p(1) .
$$

Visibly, the form $\omega$ is invariant by $S p(n+1)$. Hence, the complex contact structure $\left\{U_{i}, \omega_{i}\right\}$ on $P_{2 n+1}$ is invariant by $S p(n+1)$. Let $o$ denote the origin of the homogeneous space $P_{2 n+1}=S p(n+1) / S p(n) \times T^{1}$. Then the isotropy group $S p(n) \times T^{1}$ acts irreducibly on the hyperplane $E_{o}$ of the tangent space $T_{o} P_{2 n+1}$ defined by (4.1). Since $S p(n+1)$ is simple and $S p(n) \times T^{1}$ is the centralizer of $T^{1}$ in $S p(n+1)$, we can apply (3.4) to obtain

(5.6) Theorem. Let $E$ be the vector bundle of rank $2 n$ over $P_{2 n+1}=$ $S p(n+1) / S p(n) \times T^{1}$ defined by an invariant complex contact structure, (see $(4.1))$.

(1) Let $h$ be an $S p(n+1)$-invariant Hermitian structure in $E$ and let $g$ be an $S p(n+1)$-invariant Kähler metric on $P_{2 n+1}$. Then $(E, h)$ is an 
Einstein-Hermitian vector bundle over $\left(P_{2 n+1}, g\right)$ with irreducible holonomy group;

(2) $E$ is $H$-stable in the sense of Mumford-Takemoto for any ample line bundle $H$ over $P_{2 n+1}$.

If we apply (4.4) to this example, then

$$
c_{1}\left(P_{2 n+1}\right)=(n+1) c_{1}(F) .
$$

Hence,

$$
c_{1}(F)=2 \alpha,
$$

where $\alpha$ is the positive generator of $H^{2}\left(P_{2 n+1} ; Z\right)$. Let $H$ denote the hyperplane line bundle over $P_{2 n+1}$. Then

$$
F=H^{2} .
$$

From (4.8) we obtain a non-degenerate skew-symmetric bilinear form on $E(-1)=E \times H^{1}$. From (4.9) we obtain an isomorphism

$$
E(-1) \approx E(-1)^{*} \text {. }
$$

The vector bundle $E(-1)$ is called a null correlation bundle over $P_{2 n+1}$. From (5.6) it follows that $E(-1)$ is an Einstein-Hermitian vector bundle with irreducible holonomy group and hence is $H$-stable. (Since $c_{1}(E(-1)$ ) $=0$, the bundle $E(-1)$ admits actually an Einstein-Hermitian structure with $K=0$ ). It is not difficult verify (see Okonek-Schneider-Spindler [10]) that the total Chern class of $E(-1)$ is given by

$$
1+\alpha^{2}+\alpha^{4}+\cdots+\alpha^{n} \text {. }
$$

The fact that $E(-1)$ is $H$-stable is well known (Okonek-Schneider-Spindler [10]) and Lübke constructed in his thesis [8] an Einstein-Hermitian structure in $E(-1)$ for $n=1$.

\section{§ 6. Cotangent projective bundle over $\boldsymbol{P}_{n+1}$}

We shall consider another example of complex contact manifold. In general, let $V$ be a complex manifold of dimension $n+1$ and $T^{*} V$ its holomorphic cotangent bundle. From $T^{*} V$ we construct the cotangent projective bundle $M=P\left(T^{*} V\right)$, which is a holomorphic bundle over $V$ with fibre $P_{n}$. Then $\operatorname{dim} M=2 n+1$. Let $\omega$ be the holomorphic 1-form defined on the total space of $T^{*} V$ as follows: 


$$
\omega(X)=v(p * X) \quad \text { for } \quad X \in T_{v}\left(T^{*} V\right), \quad v \in T^{*} V,
$$

where $p: T^{*} V \rightarrow V$ is the projection. In terms of a local coordinate system $z^{0}, z^{1}, \cdots, z^{n}$ of $V$ and the induced local coordinate system $z^{0}, z^{1}, \cdots$, $z^{n}, \zeta_{0}, \zeta_{1}, \cdots, \zeta_{n}$ of $T^{*} V$, the form $\omega$ is given by

$$
\omega=\zeta_{0} d z^{0}+\zeta_{1} d z^{1}+\cdots+\zeta_{n} d z^{n} .
$$

Then as in the first example discussed in the preceding section, the 1-form $\omega$ induces a complex contact structure in $M$. Then (4.1) defines a subbundle $E$ of rank $2 n$ of the tangent bundle $T M$.

In this case, $E$ has a subbundle $E^{\prime}$ of rank $n$ consisting of vectors tangent to fibres of the fibering $M \rightarrow V$. We denote the quotient bundle $E / E^{\prime}$ of rank $n$ by $E^{\prime \prime}$. If we denote the pull-back of $T V$ to $M$ by the same symbol $T V$, then all these vector bundles over $M$ can be organized by a diagram of commutative sequences as follows:

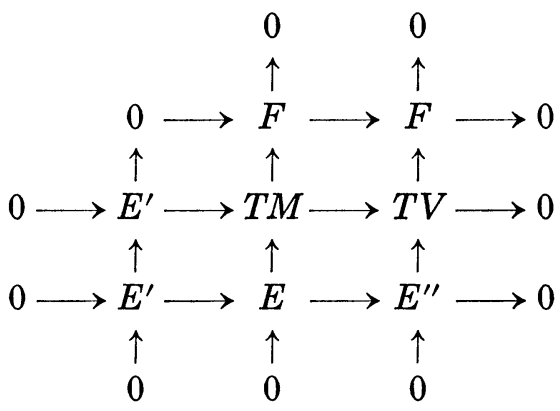

The bilinear form $\left\{d \omega_{i}\right\}: E \times E \rightarrow F$ of (4.8) induces a non-degenerate pairing

$$
E^{\prime} \times E^{\prime \prime} \longrightarrow F
$$

and an isomorphism

$$
E^{\prime \prime} \approx E^{\prime *} \otimes F
$$

Every automorphism of $V$, lifted to $T^{*} V$, leaves the 1-form $\omega$ invariant. The induced transformation of $M$ leaves the complex contact structure (defined by $\omega$ ) invariant. It leaves the vector bundles $E$ and $E^{\prime}$ also invariant and induces an automorphism of the quotient bundle $E^{\prime \prime}$.

We consider the special case where $V=P_{n+1}$. Then the action of $S U(n+2)$ on $V=P_{n+1}$ induces actions of $S U(n+2)$ on $M, E, F, E^{\prime}$ and $E^{\prime \prime}$. It is easy to verify that $S U(n+2)$ acts transitively on $M$ with the 
isotropy subgroup

$$
S(U(n) \times S U(1) \times U(1))=S U(n+2) \cap(U(n) \times U(1) \times U(1))
$$

so that

$$
M=S U(n+2) / S(U(n) \times U(1) \times U(1)) .
$$

The isotropy group is the centralizer of a torus $\left(\approx T^{2}\right)$ in $S U(n+2)$ and acts irreducibly on the fibres of $E^{\prime}$ and $E^{\prime \prime}$ at the origin of $M$. By (3.4) we have

(6.6) Theorem. Let $E^{\prime}$ and $E^{\prime \prime}$ be the vector bundles of rank $n$ over $M=P\left(T^{*} P_{n+1}\right)$ defined above.

(1) Let $h^{\prime}$ and $h^{\prime \prime}$ be $S U(n+2)$-invariant Hermitian structures in $E^{\prime}$ and $E^{\prime \prime}$, respectively. Let $g$ be an $S U(n+2)$-invariant Kähler metric on $M$. Then $\left(E^{\prime}, h^{\prime}\right)$ and $\left(E^{\prime \prime}, h^{\prime \prime}\right)$ are Einstein-Hermitian vector bundles over $(M, g)$ with irreducible holonomy group;

(2) $E^{\prime}$ and $E^{\prime \prime}$ are $H$-stable in the sense of Mumford-Takemoto for any ample line bundle $H$ over $M$.

Let $F^{\prime \prime}$ be the hyperplane line bundle over $V=P_{n+1}$. Its pull-back to $M$ will be denoted also by $F^{\prime \prime}$. Let $F^{\prime}$ be the line bundle over $M$ defined by

$$
F=F^{\prime} \otimes F^{\prime \prime}
$$

Let $\alpha^{\prime}=c_{1}\left(F^{\prime}\right), \alpha^{\prime \prime}=c_{1}\left(F^{\prime \prime}\right)$ and $\alpha=c_{1}(F)=\alpha^{\prime}+\alpha^{\prime \prime}$. Then

$$
H^{*}(M ; Z)=\left(H^{*}(V ; Z)\right)[\alpha],
$$

where the minimal equation for $\alpha$ is given by

$$
\alpha^{n+1}-c_{1}(V) \alpha^{n}+\cdots+(-1)^{n+1} c_{n+1}(V)=0 .
$$

By (6.5),

$$
E^{\prime \prime} \otimes F^{\prime \prime-1} \approx\left(E^{\prime} \otimes F^{\prime-1}\right)^{*} .
$$

The bundle $E^{\prime} \otimes F^{\prime-1}$ or its dual $E^{\prime \prime} \otimes F^{\prime \prime-1}$ is perhaps an analogue of a null correlation bundle. Their Chern classes can be computed easily. In fact, from the Euler sequence

$$
0 \longrightarrow F^{\prime \prime-1} \longrightarrow C^{n+2} \longrightarrow T V \otimes F^{\prime \prime-1}
$$

over $V=P_{n+1}$, we obtain the total Chern class of $T V \otimes F^{\prime \prime-1}$ : 


$$
c\left(T V \otimes F^{\prime \prime-1}\right)=\frac{1}{1-\alpha^{\prime \prime}} .
$$

From (6.3) we have an exact sequence

$$
0 \longrightarrow E^{\prime \prime} \otimes F^{\prime \prime-1} \longrightarrow T V \times F^{\prime \prime-1} \longrightarrow F^{\prime} \longrightarrow 0
$$

over $M$. Hence,

$$
c\left(E^{\prime \prime} \otimes F^{\prime \prime-1}\right)=\frac{1}{\left(1+\alpha^{\prime}\right)\left(1-\alpha^{\prime \prime}\right)} \cdot
$$

From (6.7) and (6.8) we obtain

$$
c\left(E^{\prime} \otimes F^{\prime-1}\right)=\frac{1}{\left(1-\alpha^{\prime}\right)\left(1+\alpha^{\prime \prime}\right)} .
$$

\section{BIBLIOGRAPHY}

[1] W. M. Boothby, Homogeneous complex contact manifolds, Proc. Symp. Pure Math., 3 (1961), Amer. Math. Soc. 144-154.

[2] - A note on homogeneous complex contact manifolds, Proc. Amer. Math. Soc., 13 (1962), 276-280.

[ 3 ] A. Borel, Kählerian coset spaces of semi-simple Lie groups, Proc. Nat. Acad. Sci. USA, 40 (1954), 1147-1151.

[4] S. Kobayashi, Remarks on complex contact manifolds, Proc. Amer. Math. Soc., 10 (1959), 164-167.

[ 5 ] — First Chern class and holomorphic tensor fields, Nagoya Math. J., 77 (1980), 5-11.

[6] - Curvature and stability of vector bundles, Proc. Japan Acad., 58 (1982), 158-162.

[ 7 ] — Differential Geometry of Holomorphic Vector Bundles, Mathematics Seminar Notes, 41 (1982), University of Tokyo. (In Japanese).

[ 8 ] M. Lübke, Hermite-Einstein-Vektorbündel, Dissertation, Bayreuth, 1982.

[9] — Stability of Einstein-Hermitian vector bundles, Manuscripta Math., 42 (1983), 245-257.

[10] C. Okonek, M. Schneider and H. Spindler, Vector Bundles on Complex Projective Spaces, Progress in Math., 3 (1980), Birkhäuser.

[11] S. Ramanan, Holomorphic vector bundles on homogeneous spaces, Topology, 5 (1966), 159-177.

[12] S. M. Salamon, Quaternionic manifolds, Symposia Math., 26 (Convegno del Maggio 1981), 1982, Ist. Naz. Alta Mat. F. Severi, 139-151, Academic Press.

[13] H. Umemura, On a theorem of Ramanan, Nagoya Math. J., 69 (1978), 131-138.

[14] H. C. Wang, Closed manifolds with homogeneous complex structure, Amer. J. Math., 76 (1954), 1-32.

[15] J. A. Wolf, Complex homogeneous contact manifolds and quaterninonic symmetric spaces, J. Math. Mech., 14 (1965), 1033-1048.

Department of Mathematics

University of California, Berkeley

Berkeley, California 94720

U.S.A. 